\title{
Response Mode and Choice Consistency: A Test of Unfolding Theory
}

\author{
BRUCE W. CARLSON \\ University of Michigan
}

\begin{abstract}
Coombs, Donnell, and Kirk (1978. Journal of Experimental Psychology, 4, 497-512), in a study of risk preferences, collected data using both pick $\frac{1}{3}$ and reject $\frac{1}{3}$ response modes. Although the preference orders derived from the two response modes were identical, the pick data contained a greater number of inconsistencies than the reject data. In the present study, predictions were derived from unfolding theory (Coombs, 1964. A theory of data. New York: Wiley) regarding the relative consistency of pick and reject response modes. An experiment performed as a test of these predictions supported the unfolding model suggesting that differences in inconsistency between response modes could be attributed to the fineness of the grid of working midpoints imposed upon the choice process by the response mode.
\end{abstract}

c. 1984 Academic Press, Inc.

Coombs (1964) distinguished between pick $k / p$ and reject $k / p$ response modes, where $k$ stimuli are either picked or rejected from a set of $p$ stimuli. Coombs $e t$ al. (1978), using lotteries in an investigation of risk-taking, reported that peoples' resultant preference scales, one obtained from a pick $\frac{1}{3}$ response mode and another obtained from a reject $\frac{1}{3}$ response mode, were virtially identical. However, they discovered that data generated in the pick task were less consistent than data generated in the reject task. Further, the greater inconsistency was related to more violations of single-peakedness ${ }^{1}$ in the preference orders constructed from pick data.

This paper derives a set of predictions from Coombs' (1964) unfolding theory regarding the relative consistency of pick and reject response modes. An experiment is reported which tests these predictions. Finally, the implications of these results for other choice models and for choice behavior in general are discussed.

This rescarch was funded by PHS training Grant GM-01231 from the National Institutes of Health and by National Science Foundation Grant BNS 78-09101. The author wishes to thank David Meyer, Jerry Cohen, Annette Leopard, and especially, Clyde Coombs for their many helpful suggestions and comments. Address reprint requests to Bruce W. Carlson, Michigan Mathematical Psychology Program, Department of Psychology, University of Michigan, 580 Union Drive, Ann Arbor, Michigan 48109.

${ }^{1}$ Preference behavior satisfies single-peakedness over a stimulus dimension when preference increases monotonically over the dimension to some ideal point after which it decreases monotonically. See Coombs and Avrunin (1977) for a discussion of necessary and sufficient conditions leading to single peakedness over a stimulus domain. 


\section{UNFOLDING THEORY (UFT)}

Unfolding theory (Coombs, 1964) is a theory of preferential choice which maps individuals and stimuli into a common space. In one dimension, the individuals, represented by ideal points, and the stimuli are mapped into a common dimension called a $J$ scale. A person's preference ordering, an $I$ scale, corresponds to the ordering of the stimuli ranked from least to greatest distance from his or her ideal point. That is, an $I$ scale represents the stimulus ordering obtained by folding a $J$ scale at an ideal point.

Unfolding theory attributes the occurrence of inconsistencies to two factors. First, Coombs (1964) postulated the existence of stimulus distributions much the same as Thurstone's (1927) discriminal dispersions. Overlap of these stimulus distributions enables one alternative to be preferred over another with probability less than one. Second, Coombs (1958) demonstrated an increase in inconsistency for bilateral pairs, stimulus pairs straddling an ideal point, relative to unilateral pairs, stimulus pairs on the same side of the $J$ scale relative to the ideal point, as a function of a variable ideal point. These two factors provide a means for predicting differences in the consistency of pick and reject data.

To be specific, let us make the following assumption:

UFT. The consistency of choice judgments increases monotonically with the distance from a person's ideal point to the working midpoints, midpoints (between pairs of stimuli) used in determining the choice.

This assumption is justified because if a variable ideal point may fall on either side of a particular midpoint, either stimulus may be preferred in experimentally independent replications of the choice. Thus, if the median point of such a distribution of ideal points falls on the midpoint, the choice probability of one stimulus over the other will be one-half except for sampling error. However, as the distribution is translated away from the midpoint, the probability of choosing the nearer stimulus cannot decrease. Instead, as the ideal point becomes further removed from the midpoint, the discrimination becomes simpler, approaching in the limit that of a unilateral comparison.

Typically, for any particular person and any one choice set, only one working midpoint will affect the choice judgment. However, by increasing the number of working midpoints, the probability that an ideal point will fall on or near a working midpoint also increases. Further, when the variability of the working midpoint distribution is high and/or the variability of the ideal point is high, then increasing the number of working midpoints increases the probability that more than one working midpoint affects preference judgments over replications of any choice set. As the number of working midpoints increases, then so should the inconsistency of the choice judgment.

Consider the case in which stimuli may be scaled unidimensionally. The working midpoints for pick $k / p$ are those between stimuli that have $k-1$ of the remaining 
$p-2$ stimuli between them in their rank order on the $J$ scale. For example, if the task is pick $1 / p$, then the working midpoints are those between adjacent stimuli, i.e., $k-1=0$. For reject $k / p$, the working midpoints are the same as those for pick $(p-k) / p$. So, for example, in the case of reject $1 / p$, the only working midpoint for the $p$ stimuli is the midpoint of the two stimuli which are furthest apart on the $J$ scale. If the stimuli are ordered alphabetically on the $J$ scale, then with just four stimuli in a choice set, say $A, B, C$, and $D$, there are three working midpoints for pick $\frac{1}{4}, A \mid B$, $B \mid C$, and $C \mid D$. However, for reject $\frac{1}{4}$ (and correspondingly pick $\frac{3}{4}$ ), there is only one, $A \mid D$.

Unfolding theory, for this example, predicts that the reject response mode will yield more consistent data because it imposes a coarser grid on the judgment process (in terms of the number of working midpoints). Figure 1 illustrates the working midpoints for pick/reject $k / 4$.

In general, for pick $k / p$ and reject $k / p$, the number of working midpoints is $p-k$ and $k$, respectively, so pick data will be less consistent than reject data if $p / 2>k$. If $p / 2=k$ then the number of working midpoints for pick and reject will be the same so unfolding theory predicts pick and reject data should be equally consistent, e.g., reject $\frac{2}{4}$ and pick $\frac{2}{4}$ in Fig. 1 . Finally, if $p / 2<k$ unfolding theory predicts pick data should be more consistent than reject data.

These considerations lead to a number of predictions:

UFT PREDiction 1. If the experimental tasks are pick $k / p$ and reject $(p-k) / p$, then the consistency of data generated by these two response modes will be equal.

Pick $k / p$ and reject $(p-k) / p$ should yield data of equal consistency, regardless of the dimensionality and configuration of the underlying space, because the working midpoints for these two response modes are identical.

UFT PReDiction 2. If the stimulus set is composed of $n$ elements embedded in a Euclidean space of $r$ dimensions, then the consistency of pick $k / p$ and reject $k / p$ data will be equal for $p \leqslant r+1$.
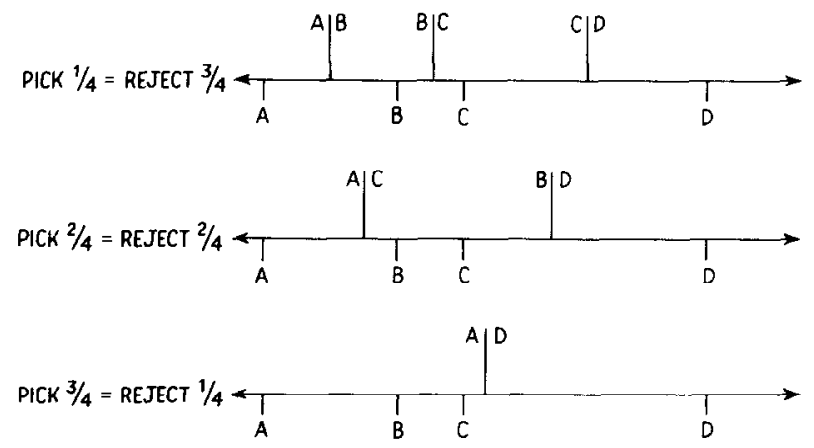

FIG. 1. Working midpoints for pick $k / 4$ and reject $k / 4$. 
The derivation of this prediction is contained in the Appendix.

The crucial point here is that unfolding theory predicts that the size of the choice sets and the dimensionality of the underlying space determine the relative consistency of pick and reject data. For example, pick $\frac{1}{3}$ data should be more inconsistent than reject $\frac{1}{3}$ data when the stimulus set may be scaled unidimensionally, but not when it may be scaled in two or more dimensions.

Certain special cases may arise. In one dimension, if an ideal point lies on a working midpoint, inconsistencies, attributable to that working midpoint, should occur regardless of whether the task is pick $\frac{1}{2}$ or reject $\frac{1}{2}$. However, for stimuli scaled in more than one dimension and $p>2$, the set of working midpoints, contributing to inconsistent choices, for any person when picking will not be the same set as when rejecting. For example, consider Fig. 2 which contains four stimuli embedded in a two-dimensional space. The lines in this space, called boundary hyperplanes, correspond to regions of equidistance between pairs of stimuli and the stimulus sequences represent preference orderings of the four stimuli. Suppose that triples of stimuli are presented to a person whose ideal point lies on the $A B$ bounding hyperplane in such a way that $A$ and $B$ are always preferred to the stimulus that completes the triple, e.g., point $P$ in Fig. 2 . If the task is pick $\frac{1}{3}$, preference judgments will be highly inconsistent when triples containing the $A, B$ pair are presented. However, for these same triples, if the task is reject $\frac{1}{3}$, the third member of the triple should be consistently rejected. Thus, for each person, inconsistencies result from the nearness of the ideal point to different sets of working midpoints when rejecting and picking.

Therefore, in 2-space, for example, even though data from pick $\frac{1}{3}$ and reject $\frac{1}{3}$ response modes should be equally consistent, there may exist a subset of subjects for whom the consistency of these two response modes will be unequal. Unfolding theory simply asserts that since the working midpoints for both tasks are the same the consistency of both response modes should be equal.

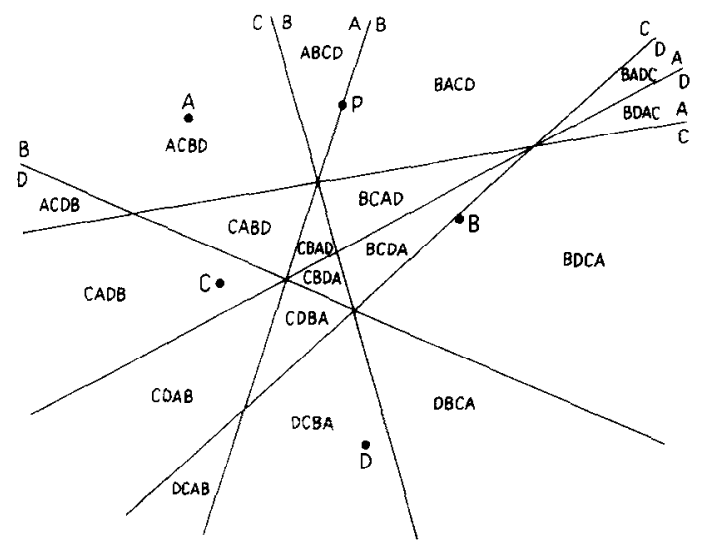

FIG. 2. Configuration of four points in two dimensions. 
Earlier it was asserted that $I$ scales are folded $J$ scales. An $I$ scale number is one more than the number of pairwise permutations required to change an $I$ scale order into the corresponding $J$ scale order. The ideal point of a person with a high- or lownumbered $I$ scale will be near one of the ends of the $J$ scale, while the ideal point of a person with a medium-numbered $I$ scale will be near the median stimulus in the $J$ scale order.

UFT PREDICTION 3. If the stimulus set may be scaled in 1 dimension, then data generated by people with high- or low-numbered $I$ scales will be more consistent than data generated by people with medium-numbered $I$ scales. This difference will increase monotonically with $p$ for reject $k / p$ (with $k$ for pick $k / p$ ).

The derivation of this prediction is contained in the Appendix.

UFT Prediction 3 follows from the fact that a greater number of bilateral pairs exists for those who fold the $J$ scale nearer the median stimulus, and more inconsistencies result from bilateral than unilateral pairs (Coombs, 1958).

The following experiment was designed as a test of these predictions.

\section{METHOD}

\section{Subjects}

Thirty-two University of Michigan students, selected from the paid subject pool and solicited through an ad placed in the campus newspaper, participated in this study. One-half were male and one-half were female. Each received $\$ 2.50 / \mathrm{hr}$ for participation.

\section{Stimuli}

Two different sets of stimuli were constructed. The types of stimuli chosen, grays and colors, were selected for two principal reasons. First, their metric properties were fairly well-known; a test of the predictions required that one of the stimulus sets could be scaled unidimensionally while the other could be scaled multidimensionally. Second, they had been used successfully in previous studies of human judgment.

The first set of stimuli, a unidimensional stimulus set, consisted of seven shades of gray from the Color-Vu series. Each $18 \mathrm{in.} \times 24 \mathrm{in} .(45.72 \mathrm{~cm} \times 50.96 \mathrm{~cm})$ gray sheet was cut into square patches measuring $45 \mathrm{~mm} / \mathrm{side}$. Each choice set consisted of two, three, or four patches mounted on a 5 in. $\times 7$ in. $(12.70 \mathrm{~cm} \times 17.78 \mathrm{~cm})$ white index card. Pairs and triples were mounted side by side while quadruples were mounted in two rows of two each. Below each patch was a number from one to four which the subject used to indicate his or her choice on a separate score sheet. To minimize variation, only a single sheet was used to construct the patches for each shade of gray. Approximately $25 \%$ of the choice sets were reordered after each session.

The second set of stimuli, a multidimensional stimulus set, consisted of seven colors from the Color-Vu series. These corresponded roughly to: red, yellow, brown, 
blue, orange, green, and violet. Choice sets were created by a procedure similar to that described above except only triples were formed.

\section{Procedure}

Each subject attended three sessions individually; each session lasted approximately $1 \mathrm{hr}$. Subjects were randomly assigned to one of two equal-sized groups. The first group judged the gray stimulus set the first two days and the color stimulus set the third. The second group judged the colors the first day and the grays the next two. All subjects participated on two consecutive days for the gray stimulus sessions. Although subjects were encouraged to attend three successive days up to three days were allowed between the gray stimulus sessions and the color stimulus session.

The two groups were each further subdivided into four equal subgroups. The first eighth performed three tasks on the first day: pick $\frac{1}{2}$, pick $\frac{1}{3}$, and pick $\frac{1}{4}$. Pick $\frac{1}{2}$ involved presenting pairs of gray color patches to each subject and instructing him or her to select that shade of gray which was "most representative of gray or closest to your ideal conception of gray." Pick $\frac{1}{3}$ and pick $\frac{1}{4}$ were identical except that choice sets consisted of triples or quadruples. Choice sets for pick $\frac{1}{2}$, pick $\frac{1}{3}$, and pick $\frac{1}{4}$ consisted, respectively, of 63 pairs, 105 triples, and 105 quadruples. For each subject, the presentation order of the 273 choice sets was randomized.

On the second day each member of this subgroup took part in the following three tasks: reject $\frac{1}{2}$, reject $\frac{1}{3}$, and reject $\frac{1}{4}$. For each choice set the subject was instructed to choose that gray which was "least representative of gray or furthest from your ideal conception of gray." Again the presentation order was randomized.

On the third day this subgroup was presented one-half of the color choice sets and asked to choose that color which was "most attractive or prettiest" (pick $\frac{1}{3}$ ). During the second half of the session the remaining color triples were presented and each subject was asked to choose that color which was "least attractive or ugliest" (reject $\left.\frac{1}{3}\right)$. Within each half session, the presentation order of the 105 triples was randomized. Throughout the entire session all subjects saw each permutation of three colors once. Each half session was balanced with respect to all pairs of stimuli. That is, each pair of colors was embedded in the same number of triples as all other pairs. The remaining seven subgroups were assigned one of the remaining permutations of the stimulus sessions: pick $1 / p$ grays for $p=2,3,4$, reject $1 / p$ grays for $p=2,3,4$, pick $\frac{1}{3}$ colors, and reject $\frac{1}{3}$ colors (with the constraints that both color tasks were on the same day and both gray sessions were on successive days).

\section{RESULTS}

\section{Construction of Preference Orders}

Preference orders were constructed using the Method of Total Vote Count (Coombs, 1964). For pick $k / p$, a count was made of the number of times each stimulus was chosen in all $p$-element choice sets. The preference order corresponded to the rank order of the stimuli from most to least frequently chosen. Since reject $k / p$ 
is numerically equivalent to pick $(p-k) / p$, preference orders for reject data were constructed using the number of times each stimulus was not rejected. For each subject, two preference orders were constructed from the color data, one for pick $\frac{1}{3}$ and one for reject $\frac{1}{3}$. For the gray data, seven preference orders were constructed for each subject, one each for pick/reject $\frac{1}{2}, \frac{1}{3}, \frac{1}{4}$, and one combining both the pick and reject data.

\section{Determination of the Number of Inconsistencies}

Once the preference orderings were constructed, the number of inconsistencies were determined. One approach is to divide in half the sum of the absolute differences between the expected frequencies obtained from the Method of Expected Matrices (Coombs, 1964) and the resultant frequencies derived from the data (e.g., Coombs $e t$ al., 1978). This method, however, only establishes a lower bound for the number of inconsistencies.

An alternative approach, used in this paper, is to treat each choice set as a set of pair comparisons. For example, for pick $\frac{1}{4}$, each set of four stimuli contains six implicit pair comparisons for which information is only obtained on three of them, i.e., between the preferred stimulus and each of the remaining stimuli in the quadruple. Thus one inconsistency results whenever one member of the choice set is chosen over a second when, according to the preference order, the second is preferred to the first. ${ }^{2}$ The number of inconsistencies for each subject for all nine scales was calculated in this manner. ${ }^{3}$ Two subjects were eliminated from analysis of the gray data ${ }^{4}$ and one from analysis of the color data $^{5}$ on the basis of substantial evidence that their responses were random.

\section{Statistical Analysis}

For each scale, the number of inconsistencies was divided by the number of pair comparisons in all choice sets from the respective pick/reject response mode(s). The analysis was performed on these normalized scores. Comparisons between pick and reject inconsistency were made according to the predictions discussed earlier. ${ }^{6}$

\footnotetext{
${ }^{2}$ For example, assume the preference order was $A B C D$. If this quadruple was presented and $B$ was chosen in pick $\frac{1}{4}$, this was counted as one inconsistency, if $C$ was chosen it was counted as two inconsistencies, and if $D$ was chosen it was counted as three.

${ }^{3}$ If two stimuli were tied in the preference order, i.e., each was chosen an identical number of times. that ordering which minimized the number of inconsistencies was chosen. Among the 352 preference orders this occurred 61 times or $17.3 \%$ of the total.

${ }^{4}$ A subject was eliminated if either every pick or every reject preference order violated singlepeakedness, and if, for either pick $\frac{1}{2}$ or reject $\frac{1}{2}$, responses to 12 or more of the 63 pairs were inappropriate. That is, the response entered on the score sheet was either a three or four when the choice set consisted of only two stimuli. Additionally, the two subjects eliminated were the most inconsistent subjects.

${ }^{5}$ The data, of the subject eliminated, contained approximately $50 \%$ more inconsistencies than the data of the next most inconsistent subject. In fact, $43 \%$ of all pairs were classified as inconsistent. Further, the differences in the frequencies of choice between the most and least preferred stimuli were only nine and five, for pick $\frac{1}{3}$ and reject $\frac{1}{3}$, respectively; the expected difference is 30 .

${ }^{6}$ All statistical tests were two-tailed.
} 
TABLE 1

Median Percent Inconsistency by Response Mode for Each Stimulus Type

\begin{tabular}{cccccc}
\hline & \multicolumn{3}{c}{ Grey Stimuli $^{a}$} & & Color Stimuli $^{b}$ \\
\cline { 2 - 4 } Response Mode & $\frac{1}{2}$ & $\frac{1}{3}$ & $\frac{1}{4}$ & & $\frac{1}{3}$ \\
\hline Pick & 2.4 & 5.5 & 5.7 & \\
Reject & 3.2 & 1.7 & 1.3 & & 8.5 \\
\hline
\end{tabular}

${ }^{a} n=30$.

${ }^{b} n=31$.

Within-subject comparisons of the differences in inconsistency between pick and reject $\frac{1}{2}, \frac{1}{3}$, and $\frac{1}{4}$ were made using the Wilcoxon signed rank sum test (see Table 1 ). ${ }^{7}$ For the gray stimulus set, pick $\frac{1}{3}$ data were more inconsistent than reject $\frac{1}{3}$ data $(T=2.58, p=.010)$ and pick $\frac{1}{4}$ data were more inconsistent than reject $\frac{1}{4}$ data $(T=3.05, p=.002)$, but no difference in inconsistency was detected for pick $\frac{1}{2}$ data and reject $\frac{1}{2}$ data $(T=-0.39, p>.500)$. For the color stimulus set, no difference in inconsistency was found between pick $\frac{1}{3}$ data and reject $\frac{1}{3}$ data $(T=0.97, p=.330)$.

A between-subject test of the third prediction of unfolding theory, that $I$ scale numbers and inconsistency should be related, was also conducted. Subjects were assigned $I$ scale numbers based on the preference ordering determined from their combined pick and reject data. They were then partitioned into two groups. The first group contained subjects with one of the first two or last two $I$ scale numbers and the second group contained the remaining subjects. Using the Wilcoxon rank sum test, subjects with extreme $I$ scale numbers $(n=22, \mathrm{Mdn}=3.7 \%)$ were found to generate more consistent data than subjects with intermediate $I$ scale numbers $(n=8$, $\operatorname{Mdn}=13.8 \%)(W=3.38, p<.001)$. That is, subjects who folded nearer the middle of the $J$ scale were less consistent than those who folded nearer the ends.

\section{Discussion}

The greater inconsistency of data generated by pick $1 / p$ methods relative to reject $1 / p$ methods reported by Coombs et al. (1978) was replicated in this study. However, this was only true under certain circumstances. Although a difference in the consistency of pick and reject data for the gray stimuli was detected when the choice sets consisted of three or four elements, no difference was observed when the choice

\footnotetext{
7 The subject eliminated from analysis of the color data had rejected first and then picked as did the two subjects eliminated from analysis of the gray data. To avoid bias in the statistical analysis due to learning effects from picking first and then rejecting or vice versa, the median normalized difference inconsistency score from the remaining subjects who had rejected and then picked was substituted for the data of the eliminated subjects for this portion of the analysis.
} 
sets contained only two elements. In addition, when the choice sets were triples, a difference in the consistency of pick and reject data was found for one type of stimulus (grays) but not for another (colors). Unfolding theory attributed this to the different underlying dimensionality of the two kinds of stimuli. The additional prediction of unfolding theory, that inconsistency was greatest for those who folded in the middle of the $J$ scale relative to those who folded in the ends of the $J$ scale, was also verified. Thus, the predictions generated by unfolding theory were all sustained.

In the succeeding sections alternative explanations for these results will be considered.

\section{Stimulus Features}

The response mode, picking or rejecting, may affect the perceived salience of the stimulus features used in the choice. For example, investigations of risky decision making have reported that, for preference judgments, people choose gambles for which the probability of obtaining the most favorable outcome is greatest. However, when asked to assign bidding or selling prices to these same gambles, people tend to prefer gambles with the largest favorable outcome (Slovic \& Lichtenstein, 1968; Lichtenstein \& Slovic, 1971, 1973; Lindman, 1971). These results indicate that the expressed preference order between two gambles is not always compatible with the order implied by their selling or buying prices. Lichtenstein and Slovic (1973) attributed this difference to a correspondence between stimulus features and task demands.

Likewise, Tversky (1977) concluded that common stimulus features weighed more heavily than distinctive features in judgments of similarity. However, for dissimilarity judgments, distinctive features were more heavily weighted than common ones.

If different tasks influence the stimulus features used in the judgment process, perhaps different demands are imposed on the subject by the pick and reject response modes which result in attention to different stimulus features. One possibility, for example, is that picking focuses attention on positive stimulus features whereas rejecting causes attention to focus on the negative features.

However, if each stimulus is defined by its value on a single dimension, as are the gray stimuli, then that dimension must be used for both picking and rejecting. It follows that pick and reject data should be equally consistent. The results indicate that that is not the case. A similar argument may be used to eliminate the possibility that people use different composition rules when picking and rejecting.

\section{Failure of Single-Peakedness}

Unfolding theory demands that preferences over a stimulus space be single-peaked. As one reviewer of this paper suggested, the failure to obtain a difference in the consistency of pick and reject data for the color stimuli may be because singlepeakedness over the color preference space is violated. Unfortunately, this experiment does not permit a test of this hypothesis. 


\section{Implications for Preferential Choice}

The advantage of reject over pick response modes has now been demonstrated using two widely disparate stimulus sets: lotteries and colors. Additional research is needed to further establish the generality of these findings and the feasibility of alternative theoretical explanations.

However, these findings suggest that, in some instances, preferential choice data should be collected using reject rather than pick response modes. This is particularly true if the size of the choice sets is large relative to both the dimensionality of the stimulus set and the number of stimuli to be selected. Unfortunately, reject methods do not require that a choice be made among the most preferred stimuli. When it is important to differentiate between the most preferred stimuli, then both response modes might be used. This strategy results in choices being made between each of the stimuli while also minimizing the number of inconsistencies.

These results also suggest that inconsistency is a poor determinant of the quality of a subject's data, i.e., quality with respect to the informativeness of the data in a test of experimental hypotheses. It is quite common to identify and eliminate subjects whose responses appear to be random. Unfortunately, perfectly consistent subjects may be equally uninformative, because their data are often easily fit theoretically, perhaps because they adopt simple decision strategies designed to minimize the effort needed in the performance of the experimental task (Slovic, Lichtenstein, \& Edwards, 1965). Indeed, these results suggest that the more informative and interesting subjects, psychologically, are moderately inconsistent. For example, in the context of unfolding theory, subjects who fold the $J$ scale near the median stimulus are the most informative since they define the quantitative $J$ scale. Yet, as these results indicate, they are also the most inconsistent.

\section{APPENDIX}

UFT PREDICTION 2. If the stimulus set is composed of $n$ elements embedded in a Euclidean space of $r$ dimensions, the consistency of pick $k / p$ and reject $k / p$ data will be equal if $p \leqslant r+1$.

Derivation. The complete permutation group of orders of $r+1$ stimuli may always be embedded in a Euclidean space of dimension $r$ (Bennett \& Hays, 1960). If $p \leqslant r+1$, then all permutations of the orders of the $p$ stimuli exist in the space. Since, for each stimulus pair, a pair of orderings exist such that each ordering contains one of the two permutations of the stimulus pair in the $k$ th and $(k+1)$ th positions in the rank order, every midpoint is a working midpoint. Therefore, pick and reject data should be equally consistent.

UFT Prediction 3. If the stimulus set may be scaled in 1 dimension, then data generated by people with high- or low-numbered $I$ scales will be more consistent than 
data generated by people with medium-numbered $I$ scales. This difference will increase monotonically with $p$ for reject $k / p$ (with $k$ for pick $k / p$ ).

Derivation. Assume the task is reject $k / p$. Let $m$ be the number of stimuli on the $J$ scale between a pair of stimuli, say $A$ and $B$. If $A \mid B$ is a working midpoint, then there exists some $p$-element subset of the $n$-element stimulus set such that $A$ and $B$ are the $k$ th and $(k+1)$ th least preferred stimuli in the subset.

Such a subset exists if two constraints on $m$ are satisfied. First, if fewer than $k-1$ stimuli lie outside the $A B$ pair on the $J$ scale, then $A$ and $B$ will always be rejected regardless of the $p$-clcment subset. Thus, $n-(m+2) \geqslant k \cdots 1$. Second, at least $p-(k+1)$ stimuli, where $k+1$ is the number of elements external to $m$, must lie between $A$ and $B$ on the $J$ scale, i.e., $m \geqslant p-(k+1)$. Therefore, if there exists some $p$-element stimulus subset such that $A \mid B$ is a working midpoint, then $p-k-1 \leqslant m \leqslant$ $n-k-1$.

Assume $k$ is fixed. Then as $p \rightarrow n, m \rightarrow n-k-1$. In other words, increasing $p$ increases the minimum number of stimuli between $A$ and $B$ on the $J$ scale that are necessary for the existence of some $p$-element stimulus subset that may contain $A$ and $B$ as the $k$ th and $(k+1)$ th least preferred stimuli. Thus, as $p$ increases only the midpoints of stimulus pairs whose members lie in opposite ends of the $J$ scale may be working midpoints. This means the working midpoints must lie nearer the center of the $J$ scale relative to all other midpoints. Therefore, people with high- or lownumbered $I$ scales will be more consistent since they fold the $J$ scale at the scale's extremities. Since as $p$ increases the working midpoints will approach the center of the scale, the distance between the ideal points of people folding at the extremes and the working midpoints increases. Thus, the data of people with high- and lownumbered $I$ scales should become more consistent with increasing $p$.

Assume pick $k / p$ is the task. Define $m$ as above. If $A \mid B$ is a working midpoint, then there exists some $p$-element subset of the stimulus set such that $A$ and $B$ are the $k$ th and $(k+1)$ th most preferred stimuli in the subset.

Such a subset exists if two constraints on $m$ are satisfied. First, $m+2 \leqslant n$ where $m+2$ includes the pair $A B$ and all stimuli internal to $A B$ on the $J$ scale. Thus, $m \leqslant n-2$. Second, if $m \leqslant k-2$ either $A$ or $B$ or both will always be picked. Thus, $m \geqslant k-1$. Therefore, if there exists some $p$-element stimulus subset such that $A \mid B$ is a working midpoint, then $k-1 \leqslant m \leqslant n-2$.

Assume $p$ is fixed. Then as $k \rightarrow n-1, m \rightarrow n-2$. As this occurs, $A$ and $B$ move, in opposite directions, to the extremities of the $J$ scale while $A \mid B$ moves to its center. This movement parallels the movement of the stimuli, for $p$ increasing, when the task is reject $k / p$. Analogous conclusions may also be drawn. That is, people with lowand high-numbered $I$ scales will generate data more consistent than those with medium-numbered $I$ scales. Also, as $k$ increases the consistency of the judgments increases for those people who fold the $J$ scale at its extremes. 


\section{REFERENCES}

BenNetT, J. F., \& Hays, W. L. (1960). Multidimensional unfolding: Determining the dimensionality of ranked preference data. Psychometrika, 25, 27-43.

Coombs, C. H. (1958). On the use of inconsistency of preferences in psychological measurement. Journal of Experimental Psychology, 55, 1-7.

Coombs, C. H. (1964). A theory of data. New York: John Wiley.

Coombs, C. H., \& Avrunin, G. S. (1977). Single-peaked functions and the theory of preference. Psychological Review, 84, 216-230.

Coombs, C. H., Donnell, M. L., \& KirK, D. B. (1978). An experimental study of risk preference in lotteries. Journal of Experimental Psychology: Human Perception and Performance. 4. 497-512.

Lichtenstein, S., \& SLovic, P. (1971). Reversals of preference between bids and choice in gambling decisions. Journal of Experimental Psychology, 89, 46-55.

Lichtenstein, S., \& Slovic, P. (1973). Response-induced reversals of preference in gambling: An extended replication in Las Vegas. Journal of Experimental Psychology, 101, 16-20.

Lindman, H. R. (1971). Inconsistent preferences among gambles. Journal of Experimental Psychology, 89, 390-397.

Slovic, P., \& Lichtenstein, S. (1968). Relative importance of probabilities and payoffs in risk taking. Journal of Experimental Psychology Monograph, 78, 1-18.

Slovic, P.. Lichtenstein, S., \& Edwards, W. (1965). Boredom-induced changes in preferences among bets. American Journal of Psychology, 78, 208-217.

Thurstone, L. L. (1927). A law of comparative judgment. Psychological Review, 34, 273-286.

TVersky. A. (1977). Features of similarity. Psychological Review, 84, 327-352.

ReCEIVED: August 1, 1983 